\title{
Camptocormia treated with bilateral pallidal stimulation
}

\author{
Case report
}

\author{
DiPankar Nandi, M.B.B.S., M.Chir., Simon Parkin, M.R.C.P., Richard Scott, Ph.D., \\ Jonathan L. Winter, Carole Joint, R.G.N., RalPh Gregory, F.R.C.P., \\ JoHn STEIN, F.R.C.P., AND TIPU Z. AZIZ, F.R.C.S.
}

University Laboratory of Physiology, University of Oxford; Departments of Neurology, Neurosurgery, and Neuropsychology, Radcliffe Infirmary, Oxford; and Department of Neurology, Imperial College School of Medicine, London, United Kingdom

\begin{abstract}
The authors report the neurological, neurophysiological, and neuropsychological effects of using chronic bilateral pallidal high-frequency deep brain stimulation (DBS) in a case of disabling camptocormia.

Deep brain stimulation electrodes were implanted stereotactically to target the globus pallidus internus (GPi) bilaterally. Local field potentials (FPs) were recorded using the DBS electrodes and concurrent abdominal flexor electromyography (EMG) potentials during camptocormic episodes. Videotaped assessments of the movement disorder and neuropsychological evaluation before implantation and at 6 months after initiation of pallidal stimulation were recorded.

There was significant functional improvement following chronic pallidal stimulation, and some improvement was noted in neuropsychological scores. The GPi FPs showed temporal correlation with EMG-recorded rectus abdominis potentials. There were no treatment-related adverse effects.

The authors have found that chronic pallidal stimulation was safe and offered functional benefit in this severely disabling condition. The physiological studies may help further the understanding of the pathophysiology of this rare entity.
\end{abstract}

\section{KEY WORDS • camptocormia - pallidal stimulation • field potential • deep brain stimulation}

Camptocormia is a rare disorder involving involuntary truncal flexion induced by sitting or standing. ${ }^{28}$ It was reported as a psychogenic illness occurring among soldiers in World Wars I and II, ${ }^{9,29}$ and was subsequently regarded as a psychiatric illness. ${ }^{2,20}$ Recently it has been reported to occur in association with various other neurological conditions, ${ }^{15,23,35,37}$ including idiopathic Parkinson disease. ${ }^{5,6}$ In its severest manifestations patients are literally "doubled over" which renders most normal activities impossible or difficult. In some reported cases the disorder has been responsive to electrotherapy or to corticosteroid medications, whereas in others the disorder is refractory to all the various attempted treatment strategies. ${ }^{10}$

Chronic high-frequency DBS has been shown to improve the functional status in a number of movement disorders of varied causes. ${ }^{3,13,14,24}$ These include disorders in which the clinical manifestations are similar to those seen in camptocormia, especially conditions such as focal dystonia. ${ }^{3,11,14,27,33}$ With the development of relatively safe, image-guided stereotactic, nondestructive chronic im-

Abbreviations used in this paper: DBS = deep brain stimulation; $\mathrm{EMG}=$ electromyography; $\mathrm{FP}=$ field potential; $\mathrm{GPi}=$ globus pallidus internus; $M R=$ magnetic resonance. planted brain stimulation procedures in recent years, ${ }^{7}$ it may be scientifically and clinically valid to attempt to apply the principles and techniques of stereotactic functional neurosurgery to such a severely disabling and intractable entity as camptocormia. We have studied in detail a case of camptocormia neurologically, neurophysiologically, and neuropsychologically and present the beneficial effects of bilateral DBS of the medial GPi.

\section{CASE REPORT}

History. This 39-year-old man was referred to our clinic, which specializes in the management of movement disorders, for consideration of neurosurgical intervention to treat disabling episodes of truncal flexion. In June 1995, 4 years prior to referral, he had developed involuntary movements of the trunk, face, and limbs. Neurological examination demonstrated no underlying disease, and MR imaging of the brain revealed normal findings. His psychiatric history (ICD 10) was of generalized anxiety disorder (F41.1), emotionally unstable personality disorder (F60.3), and recurrent depressive disorder (F33). An initial diagnosis of tardive dyskinesia was made in view of prior treatment with neuroleptic medications during ad- 
missions for deliberate self-harming behavior. These included haloperidol, droperidol, chlorpromazine, and lithium. Further neuroleptic therapy was avoided, and by 1997 there had been gradual improvement in the facial and limb movements, but he continued to experience disabling involuntary flexure of the trunk. Treatment with propanolol, sodium valproate, clonazepam, tetrabenazine, and clozapine had no effect.

Examination. In 1999 he underwent evaluation in our clinic. No neurological deficits were demonstrated while he lay down; however, sitting or standing provoked involuntary flexure of the trunk beyond the horizontal plane. When he attempted to correct this, he provoked a bobbing movement and severe lumbar pain (Videotape segment 1). This combination of posture-related truncal flexure and pain is often described as camptocormia (in Latin, "bent spine") in the few cases reported in literature. 5,10,21 A diagnosis of camptocormia was made accordingly.

Click here to view videotape segment 1: Preoperative assessment performed in October 1999. The patient has a normal posture supine but is unable to maintain a sitting position without using his arms. When standing or walking, he develops severe truncal flexure that cannot be overcome. (75 MB)

In view of the severely disabling nature of his disorder and its resistance to all medical therapy, a decision was made to implant bilateral GPi stimulators. We based this decision on reports of different kinds of focal dystonias, complex dyskinesias (both parkinsonian and nonparkinsonian), tardive dystonia, and some cases of generalized dystonia treated successfully by pallidal intervention. . $^{3,14}$, 27,30,31,33,34 Informed consent was obtained.

Operation. In October 2000 he underwent computerized tomography/MR imaging-guided stereotactic implantation of bilateral DBS electrodes targeted to the anteroposterior segment of GPi, using the Radionics Image Fusion and Stereoplan. ${ }^{25}$ The electrodes were initially externalized to test the effects of stimulation. Although no benefit was seen initially, 4 days after stimulation was initiated he reported a reduction in the effort required to maintain a sitting position. A dual-channel implantable pulse generator was subsequently connected and situated subcutaneously for chronic stimulation. We obtained MR images postoperatively to confirm the site of placement ofthe DBS electrodes (Fig. 1).

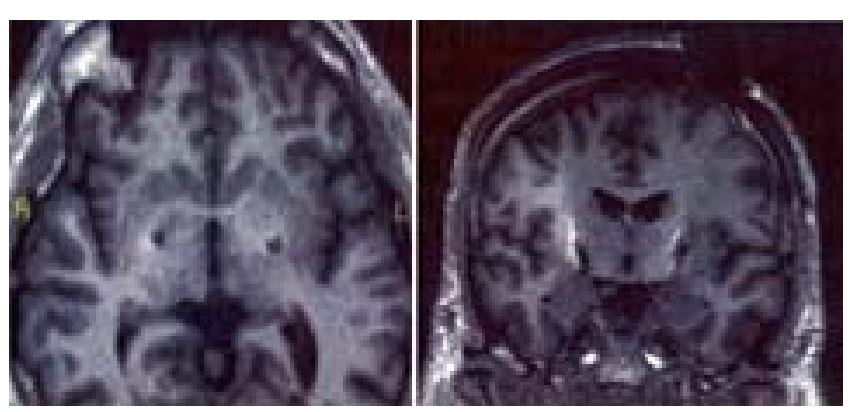

Fig. 1. Postoperative axial (left) and coronal (right) $\mathrm{T}_{1}$ weighted MR images. The electrodes are identified by areas of hypointensity surrounded by a hyperintense "flare." Both electrodes are straddling the GPi, one on each side.

\section{SOURCES OF SUPPLIES AND EQUIPMENT}

We obtained the pulse generator (Kinetra) from Medtronics (Minneapolis, MN). The Fusion and Stereoplan system was acquired from Radionics (Burlington, MA). Conmed (Rome, NY) manufactured the Neotrode Ag$\mathrm{AgCl}$ electrodes. The amplifier (1902) was purchased from CED (Cambridge, UK). Off-line analysis was performed using Matlab software produced by Mathworks, Inc. (Natick, MA). Neil Hoggarth (Laboratory of Physiology, Oxford, UK) developed the in-house software.

\section{RESULTS}

\section{Clinical Assessment}

After 1 month of chronic pallidal stimulation, the patient no longer suffered from sudden "spasms" of truncal flexure, allowing him to eat in a normal position. At the latest fol low-up examination, 6 months postoperatively, he was able to stand normally and walk in an upright position for approximately $50 \mathrm{ft}$. He was far more independent and was able to go to places such as the local pub and shops with minimal use of a cane. In addition, he can sit upright without undue effort to maintain his position (Videotape segments 2 and 3). He felt some heaviness around the neck initially in the first few weeks after initiation of stimulation; this has since subsided. He experienced no other adverse neurological effects following the pallidal stimulation. Switching off the pulse generator results in reappearance of his truncal flexion jerks within a variable period ranging from 2 to 12 hours.

Click here to view videotape segment 2: Assessment performed 3 months postoperatively (January 2001). After implantation of bilateral GPi stimulators, the patient's symptoms gradually improved. He can sit upright without much effort and can stand upright for short periods of time. When walking a short distance, his posture is more normal, with intermittent truncal flexure. Assessment performed 7 months postoperatively (May 2001). Additional improvement is apparent-he requires no effort to sit upright, stands upright for longer periods, and walks with less difficulty while experiencing fewer episodes of truncal flexure. (81 MB)

\section{Neurophysiological Assessment}

Local FPs were recorded using the externalized DBS leads placed in the GPi 2 days postoperatively. Simultaneous EMG recording of the contra- and ipsilateral rectus abdominis was performed using neonatal surface disc electrodes. Signals were sampled at $250 \mathrm{~Hz}$, amplified by 1000, filtered below $100 \mathrm{~Hz}$, digitized, and continuously displayed on-line with an adjustable window. We used inhouse developed software. Records were obtained at rest and after inducing the myoclonic-like abdominal contractions by asking the patient to stretch his arms forward or to try to stand. Off-line analysis of data was performed. During the analysis, particular attention was paid to potentials at frequencies below $20 \mathrm{~Hz}$.

Analysis of the FP and the corresponding EMG data revealed that there was a clear temporal correlation between the basal ganglia potentials recorded from the medial GPi and the muscular electrical bursts from the rectus abdominis corresponding to the myoclonic-like jerks (Fig. 


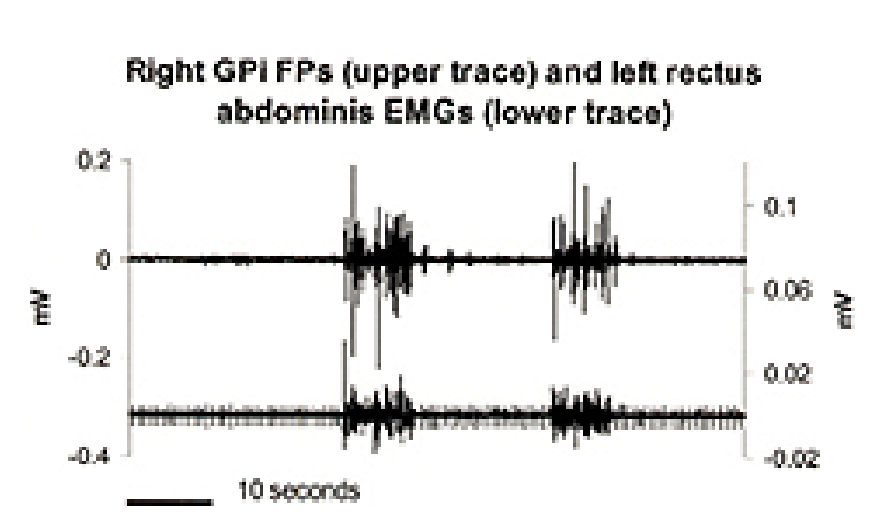

Fig. 2. Raw traces showing the FPs recorded in the right GPi and the concurrent trace from the left rectus abdominis EMG. The patient had myoclonic-like abdominal flexion at 25 and 55 seconds.

2). Fast Fourier transforms were derived to display the power spectra of the FP and EMG data (Figs. 3 and 4). The relationship between the two signals showed significant strength of correlation, or coherence-that is, the mathematical measure of correlation that we used-in the 0.5 to $3.5-\mathrm{Hz}$ range (Fig. 5). The phase relationship between the brain and muscle signals in this frequency range suggests that the GPi signals affect the muscle (Fig. 6).

\section{Neuropsychological and Functional Outcome}

We used routine preoperative Oxford Movement Disorder Group neuropsychological evaluation protocol (described in detail elsewhere ${ }^{30}$ ), which is composed of three components: 1) a clinical interview screening for any disturbances in mood, appetite, sleep, sensory-perceptual function, intellect, and thought, as well as questioning expectations of surgery, psychosocial circumstances, and any psychiatric history; 2) questionnaire measures of generic quality of life and psychological symptoms; and 3) a comprehensive psychometric assay of cognitive functions (that is, intelligence quotient, speech-language, spatial, memory, attention-executive, and speed of processing functions) (Table 1). ${ }^{1,17,26,30,32,36}$

Preoperative assessment revealed that the patient's neu-

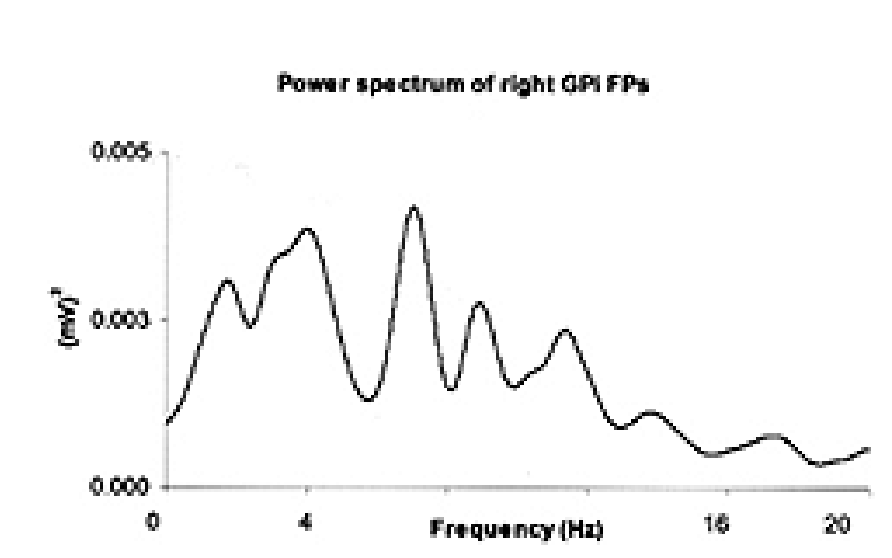

Fig. 3. Power spectrum in the frequency domain of the FPs recorded in the right GPi. The data have been filtered at below $20 \mathrm{~Hz}$.

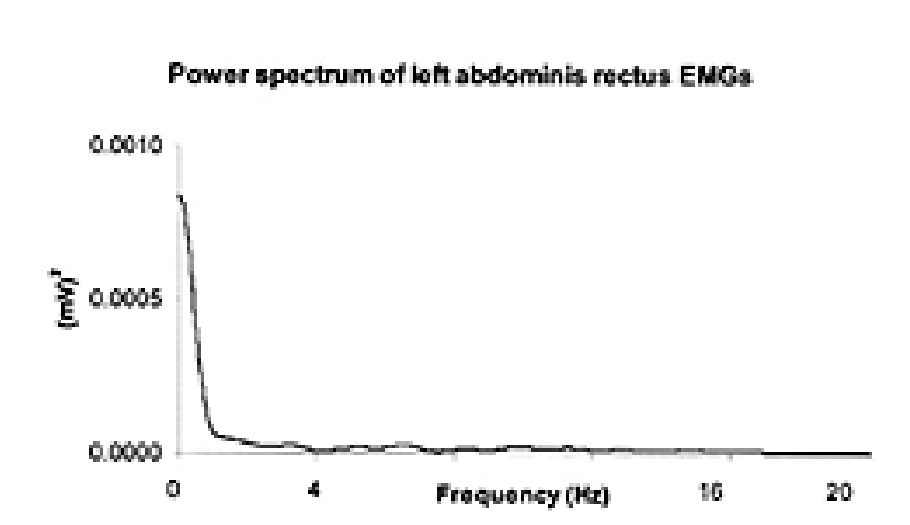

Fig. 4. Power spectrum of the left rectus abdominis EMG potentials. Only frequencies below $20 \mathrm{~Hz}$ are represented.

ropsychological profile was reasonably well preserved, although his performance on a number of tests (RSPM, phonemic fluency, story and figure recall, and recognition memory) was one standard deviation below his predicted premorbid level of ability. There was more marked and selective impairment demonstrated on tests of attentionexecutive function. His Total Percentage Disability rating on the Functional Limitations Profile (the United Kingdom version of the Sickness Impact Profile-a generic measure of quality of life ${ }^{4}$ was high $(23 \%)$, and his score (13) was at a level of "caseness" ( $>8-10$; that is, where further clinical investigation might be indicated) on the anxiety subscale of the Hospital Anxiety and Depression Scale (Table 1). Postoperative assessment at 6 months revealed clear improvements in the patient's scores in almost all cognitive domains, particularly in tests sensitive to memory/new learning ability, speed of information processing, and attention (Table 1).

\section{DISCUSSION}

\section{Clinical Outcome}

In this case the neurological problem was complex with essentially two factors contributing to the patient's clinical presentation. The background of serious psychiatric disor-

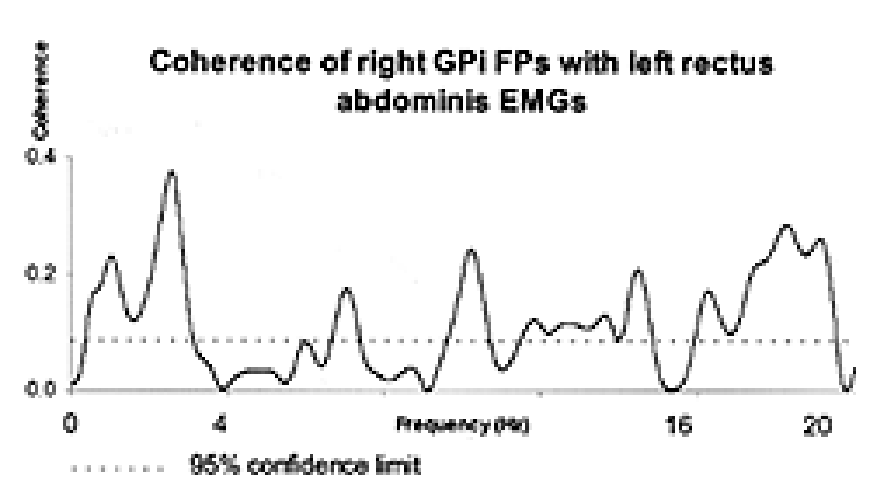

Fig. 5. Graph showing the coherence between the right GPi FPs and the left rectus abdominis EMG potentials. It shows significant coherence between the brain and muscle activity in the 0.5 to $3.5-\mathrm{Hz}$ range. 


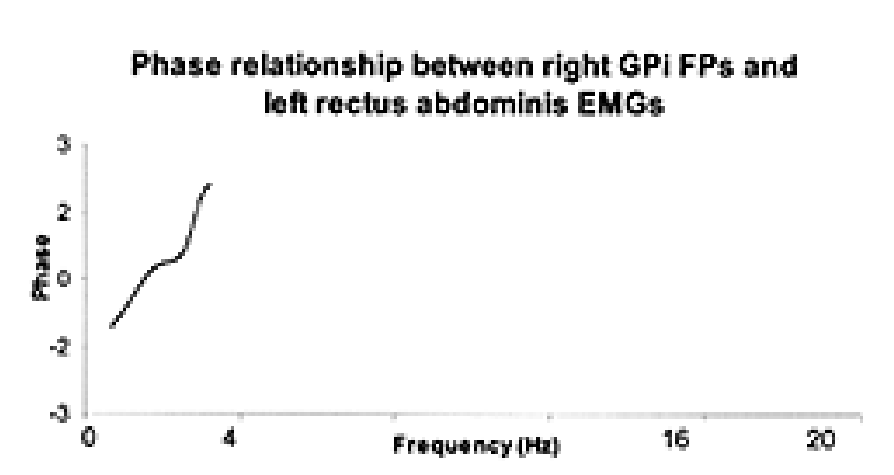

Fig. 6. Graph plotting the phase between the GPi FPs and the corresponding rectus abdominis EMG potentials in the coherent frequency range. The curve suggests that the brain signals determine the muscle activity.

der, with subsequent neuroleptic-induced tardive dyskinesia, was one factor that led to our patient's disability. These symptoms, however, had been in abeyance for the last few years, as seen in his preoperative neuropsychological profile (Table 1). The predominant feature of his present motor disability, which had such a profound debilitating effect affecting his daily life, was the camptocormic restriction on his posture and gait. In recent times camptocormia has been described as a form of segmental dystoni $^{28}$ and has also been proposed as an extreme manifestation of parkinsonian rigidity or dystonia. ${ }^{5}$ It has been shown that pallidal stimulation improves a wide variety of dystonic conditions, ${ }^{27,33,34}$ as well as parkinsonian rigidity and dyskinesias. ${ }^{7,31}$ Because our patient's symptoms were refractory to medical therapy and were severely disabling, it seemed scientifically reasonable to offer him pallidal stimulation.

In this case the treatment-related benefit was not immediate, occurring gradually over the next 6 months. This has been reported in other cases of dystonia. ${ }^{12,27,33}$ As such, it argues against a major placebo contribution to the response. Perhaps this supports other reports in which the authors propose that camptocormia is a form of segmental dystonia. ${ }^{28}$ Apart from the motor improvement in posture

TABLE 1

Preoperative and postoperative neuropsychological test scores*

\begin{tabular}{|c|c|c|}
\hline Test & Preop Scores & Postop Scores \\
\hline \multicolumn{3}{|l|}{ overall intellectual ability } \\
\hline National Adult Reading Test & 105 & \\
\hline Standard Progressive Matrices IQ & 91 & 105 \\
\hline \multicolumn{3}{|l|}{ speech \& language skills } \\
\hline Phonemic Verbal Fluency & 31 & 37 \\
\hline Categorical Verbal Fluency & 17 & 19 \\
\hline Boston Naming (no. of correct answers) & 37 & 36 \\
\hline speed \& capacity of language processing ( $\mathrm{z}$ score) & 0.44 & 1.56 \\
\hline speech articulation rates & 10.8 & 11 \\
\hline \multicolumn{3}{|l|}{ attention-executive skills } \\
\hline Digit Span (forward/backward) & $6 / 4$ & $8 / 5$ \\
\hline \multicolumn{3}{|l|}{ CANTAB } \\
\hline spatial span (z score) & -0.77 & -0.77 \\
\hline RVIP "A" Prime & -2.7 & -1.2 \\
\hline SOC (minimum move solutions) & -1.2 (raw score $6 / 12$ ) & -0.63 (raw score $8 / 12$ ) \\
\hline ID/ED (stages reached; ED-shift $=8$ ) & -1.8 (raw score $7 / 9)$ & -1.8 (raw score $7 / 9$ ) \\
\hline \multicolumn{3}{|l|}{ spatial skills } \\
\hline Judgement of Line Orientation & 24 & 28 \\
\hline copy of MCG Complex Figure & 31 & 34 \\
\hline \multicolumn{3}{|l|}{ memory/new learning } \\
\hline \multicolumn{3}{|l|}{ Recognition Memory Test } \\
\hline words & -0.33 & 0.66 \\
\hline faces & -0.66 & -0.33 \\
\hline \multicolumn{3}{|l|}{ AMIPB Story Recall } \\
\hline immediate & -0.89 & 0.59 \\
\hline delayed & -0.94 & 0.92 \\
\hline \multicolumn{3}{|l|}{ MCG Complex Figure Recall } \\
\hline immediate & 13 & 20.5 \\
\hline delayed & 12.5 & 17.5 \\
\hline \multicolumn{3}{|l|}{ questionnaire measures } \\
\hline FLP disability (total \%) & 23 & 23 \\
\hline \multicolumn{3}{|l|}{ HADS } \\
\hline anxiety & 13 & 14 \\
\hline depression & 3 & 2 \\
\hline
\end{tabular}

* The National Adult Reading Test and the Standard Progressive Matrices IQ have control mean scores of $100 \pm 15$ (standard deviation); all other values represent raw scores unless otherwise noted. Abbreviations: AMIPB = Adult Memory and Information Processing Battery; CANTAB = Cambridge Neuropsychological Test Automated Battery; ED and ID = Extradimensional/Intradimensional Set Shifting task (a decomposition of the Wisconsin Card Sorting test); FLP = Functional Limitations Profile; HADS = Hospital Anxiety and Depression Scale; MCG = Medical College of Georgia; RVIP = rapid visual information processing (a continuous performance test of sustained vigilance); SOC $=$ the Stockings of Cambridge (an analog of the Tower of London test of planning). 
and gait (Videotape segments 1-3), we also documented improvement in almost all cognitive domains, particularly in tests sensitive to memory/new learning ability, speed of information processing, and attention (Table 1). This finding reinforces the current opinion that camptocormia is not simply a motor disorder. Another important observation that refutes the likelihood of a beneficial response being due to a placebo effect was the variable period of time for the camptocormia-related physical symptoms to recur after stimulation was switched off. After 1 month of DBS, the symptoms recurred within 2 hours of ceasing stimulation. Following 6 months of DBS, it took an overnight period for the dystonic movements to manifest after stimulation was stopped. This variability has been reported in cases in which pallidal stimulation was used to treat other forms of dystonia and has been ascribed to plastic changes or to reorganization of the somatosensory input to the GPi neurons. ${ }^{33}$

\section{Electrophysiological Monitoring}

Field potential recording allows sampling of abnormal synchronous discharge in a population of neurons as opposed to the single-unit recording more commonly used in functional stereotactic neurosurgery. ${ }^{18}$ We believe this permits better understanding of abnormal electrical activity in a functionally cohesive subcortical area in patients with a pathological condition. This is especially true if there is a concurrent recording of abnormal electrical activity from related peripheral tissue - for example, EMB-detected tremor from corresponding muscle groups ${ }^{18,22}$ or cortical electrical activity. ${ }^{19}$ We have found that FP recording has improved our ability to target effective sites for chronic DBS. ${ }^{18,22}$ Highlighted by this case is the importance of monitoring of FPs from the deep brain nuclei with simultaneous recording of relevant EMG parameters, to achieve accurate placement of the stimulating electrodes. We found that the brain potentials and the corresponding EMG phenomena displayed a strong temporal correlation, and this was matched by the clinical response observed in the patient. The phase relationship between the basal ganglia and affected muscle electrical activity sheds some light on the possible pathophysiological pathway of this abnormal movement. This along with the results of the coherence study, suggests that abnormal synchronous activity in the medial GPi is closely related to the involuntary truncal flexion and perhaps drives this action. This observation supports the case for an association between GPi stimulation and clinical benefit in this patient. Other authors have also commented on the electrophysiological link with GPi activity in cases of dystonia. ${ }^{16}$

\section{CONCLUSIONS}

We have successfully treated a complex case of camptocormia by administering pallidal stimulation. We also explored the electrophysiological link between the affected muscles and the GPi in camptocormia. We hope this will add to the existing body of knowledge concerning this condition as well as to the role of pallidal stimulation in dystonic disorders in general.

\section{References}

1. American Psychiatric Association: Diagnostic and Statistical Manual of Mental Disorders: DSM-IV, ed 4. Washington DC: American Psychiatric Association, 1994

2. Ballenger JC: A case of camptocormia occurring in psychotherapy. J Nerv Ment Dis 162:291-294, 1976

3. Benabid AL, Benazzouz A, Hoffmann D, et al: Long-term electrical inhibition of deep brain targets in movement disorders. Mov Disord 13 (Suppl 3):119-125, 1998

4. Bergner M, Bobbitt RA, Carter WB, Gilson BS: The Sickness Impact Profile: development and final revision of a health status measure. Med Care 19:787-805, 1981

5. Djaldetti R, Mosberg-Galili R, Sroka H, et al: Camptocormia (bent spine) in patients with Parkinson's disease-characterization and possible pathogenesis of an unusual phenomenon. Mov Disord 14:443-447, 1999

6. Garcia de Yebenes J, Garcia Ruiz-Espiga P: Descripcion princeps de la camptocormia en la enfermedad de Parkinson por Francisco de Zurbaran a mediados del siglo XVII. Neurologia 15:265-266, 2000

7. Ghika J, Villemure JG, Fankhauser H, et al: Efficiency and safety of bilateral contemporaneous pallidal stimulation (deep brain stimulation) in levodopa-responsive patients with Parkinson's disease with severe motor fluctuations: a 2-year follow-up review. J Neurosurg 89:713-718, 1998

8. Gomez EA, Drooby AS: Camptocormia in a case of manicdepressive disorder. Psychosomatics 28:592, 594-595, 1987

9. Hurst AF: The bent back of soldiers. BMJ 2:621-623, 1918

10. Karbowski K: The old and the new camptocormia. Spine 24: 1494-1498, 1999

11. Krack P, Vercueil L: Review of the functional surgical treatment of dystonia. Eur J Neurol 8:389-399, 2001

12. Krauss JK, Pohle T, Weber S, et al: Bilateral stimulation of globus pallidus internus for treatment of cervical dystonia. Lancet 354:837-838, 1999

13. Kumar R, Lozano AM, Sime E, et al: Comparative effects of unilateral and bilateral subthalamic nucleus deep brain stimulation. Neurology 53:561-566, 1999

14. Lang AE: Surgical treatment of dystonia. Adv Neurol 78: 185-198, 1998

15. Laroche M, Delisle MB, Aziza R, et al: Is camptocormia a primary muscular disease? Spine 20:1011-1016, 1995

16. Lenz FA, Suarez JI, Metman LV, et al: Pallidal activity during dystonia: somatosensory reorganisation and changes with severity. J Neurol Neurosurg Psychiatry 65:767-770, 1998

17. Lezak MD: Neuropsychological Assessment, ed 3. New York: Oxford University Press, 1995

18. Liu X, Hayward G, Ford-Dunn H, et al: Intraoperative recording of field potentials for assisting localisation of the subthalamic nucleus. Acta Neurochir 142:1204, 2000 (Abstract)

19. Marsden JF, Ashby P, Limousin-Dowsey P, et al: Coherence between cerebellar thalamus, cortex and muscle in man: cerebellar thalamus interactions. Brain 123:1459-1470, 2000

20. Massa E, Slater CB: Camptocormia and depression: a case report. Mil Med 154:352-355, 1989

21. Miller RW, Forbes JF: Camptocormia. Mil Med 155:561-565, 1990

22. Nandi D, Liu X, Bain P, et al: Electrophysiological confirmation of the zona incerta as a target for surgical treatment of disabling involuntary arm movements in multiple sclerosis: use of local field potentials. J Clin Neurosci (In press)

23. Nieves AV, Miyasaki JM, Lang AE: Acute onset dystonic camptocormia caused by lenticular lesions. Mov Disord 16: 177-180, 2001

24. Pahwa R, Lyons K, Koller WC: Surgical treatment of essential tremor. Neurology 54 (11 Suppl 4):S39-S44, 2000

25. Papanastassiou V, Rowe SJ, Scott R, et al: Use of the Radionics 
Image Fusion $^{\mathrm{TM}}$ and Stereoplan ${ }^{\mathrm{TM}}$ programs for target localization in functional neurosurgery. J Clin Neurosci 5:28-32, 1998

26. Parker ES, Eaton EM, Whipple SC, et al: University of Southern California Repeatable Episodic Memory Test. J Clin Exp Neuropsychol 17:926-936, 1995

27. Parkin S, Aziz T, Gregory R, et al: Bilateral internal globus pallidus stimulation for the treatment of spasmodic torticollis. Mov Disord 16:489-493, 2001

28. Reichel G, Kirchhofer U, Stenner A: Kamptokormia—eine segmentale Dystonie: Vorschlag einer neuen Definition fur eine alte Krankheit. Nervenarzt 72:281-285, 2001

29. Sandler SA: Camptocormia: a functional condition of the back in neurotic soldiers. War Medicine 8:36-45, 1945 (Reference unverified)

30. Scott R, Gregory R, Hines N, et al: Neuropsychological, neurological and functional outcome following pallidotomy for Parkinson's disease. A consecutive series of eight simultaneous bilateral and twelve unilateral procedures. Brain 121:659-675, 1998

31. Siegfried J, Lippitz B: Bilateral chronic electrostimulation of ventroposterolateral pallidum: a new therapeutic approach for alleviating all parkinsonian symptoms. Neurosurgery 35: 1126-1130, 1994

32. Thompson LL, Heaton RK: Comparison of different versions of the Boston Naming Test. Clin Neuropsychol 3:184-192, 1989
33. Tronnier VM, Fogel W: Pallidal stimulation for generalized dystonia: Report of three cases. J Neurosurg 92:453-456, 2000

34. Trottenberg T, Paul G, Meissner W, et al: Pallidal and thalamic neurostimulation in severe tardive dystonia. J Neurol Neurosurg Psychiatry 70:557-559, 2001

35. Van Gerpen JA: Camptocormia secondary to early amyotrophic lateral sclerosis. Mov Disord 16:358-360, 2001

36. Woodard JL, Benedict RH, Roberts VJ, et al: Short-form alternatives to the Judgment of Line Orientation Test. J Clin Exp Neuropsychol 18:898-904, 1996

37. Zwecker M, Iancu I, Zeilig G, et al: Camptocormia: a case of possible paraneoplastic aetiology. Clin Rehabil 12:157-160, 1998

Manuscript received October 22, 2001.

Accepted in final form January 15, 2002.

This work was supported by grants from the Norman Collisson Foundation and the Medical Research Council, Untied Kingdom. Address reprint requests to: Tipu Z. Aziz, F.R.C.S., Department of Neurosurgery, Radcliffe Infirmary, Woodstock Road, Oxford, OX2 6HE, Untied Kingdom. email: tipu.aziz@ physiol.ox.ac.uk. 